\title{
Design of Non-contact Hand Shape Verification Method
}

\author{
Liu Yingxuan,a \\ ${ }^{1}$ School of Mechanical Engineering, Baicheng Normal University, Jilin, Baicheng, 137000, China \\ a414367074@qq.com
}

Keywords: Hand shape verification, Image preprocessing, Moment invariants, Feature extraction, SVM.

\begin{abstract}
A new method for personal identification based on geometric invariant moment is presented. Firstly image processing including binary processing and segment of hand silhouette are used, and then translation and scale normalization algorithms are implemented on the palms and fingers image. After that the geometric moment characteristics of image are extract, and then the feature vectors composed of seven moment invariants is obtained. At last, support vector is achieved by training 100 images data in images database, 15 testing image were selected randomly to verify validity and feasibility of algorithms. Experimental results indicate that the accuracy of hand shape identification is $93 \%$. The new method of extracting hand shape geometric moment as characteristic matrix is easy to realize with characteristic of high utility and accuracy, and solve the problem of translation, rotation and scaling during the image acquisition process without positioning aids, and especially for development and application of the portable embedded devices.
\end{abstract}

\section{Introduction}

Compared with other biological features, hand-based identification technology has the following advantages: the requirements of the image acquisition device is low, the hand processing algorithm is relatively simple and the fastest authentication speed. At present, hand authentication has become an important part of biometric identification technology, and has received more and more attention ${ }^{[1]}$. In many occasions where accuracy is not very high, identity authentication technology has a wide range of application prospects, such as the company or school attendance, exam candidates before the certification. There are two kinds of traditional hand matching methods. Based on point pattern matching and feature vector matching, the former has a small error rate, but the calculation and rejection rate is relatively large, and there are very strict requirements for the position and direction of the hand ${ }^{[2]}$. Which can be used to calculate the shape and shape of the hand shape to distinguish the different hand shape, the method is simple to calculate the amount of small, fast authentication, but the error rate is high, and can not solve the problem of finger translation, rotation and scaling, poor $\operatorname{rod}^{[3]}$. Yuan Weichi et al. proposed a hand-shaped recognition method based on the relative length of the finger, which is identified by the eigenvector of the relative length between the fingers. The results show that the recognition rate can reach $78.52 \%{ }^{[4]}$. China University of Science and Technology, such as care and other people proposed a template matching based on the hand-shaped authentication algorithm, but also get better recognition results, but the need to add the stage of the pretreatment correction step $^{[5]}$.

In this paper, a non-contact hand-shaped image acquisition and processing method for non-locating bolt is proposed. The collected image is preprocessed and normalized, and the geometric invariant moments are extracted as a set of eigenvalues of hand shape feature recognition. Because of the problem of image translation, rotation and scaling caused by the removal of the positioning bolt, the training and classification of the collected hand image are supported by the support vector machine. The experiment proves that the method has non-contact, fast and accurate classification process. Strong and so on. 


\section{Geometric moment definition}

Geometric moment is a method based on statistical analysis. In 1962, MKHu first proposed the geometrical moment theory of image recognition and proved that the translation, scaling and rotation of the proposed seven groups were unchanged ${ }^{[6]}$. In this paper, the use of geometric moments for feature extraction, and applied to the hand recognition to a good solution to the hand shape recognition process of hand rotation scaling and scale transformation of the problem, so that hand recognition system has a good robustness. The lower order moment mainly describes the overall characteristics of the image, such as area, spindle, direction angle, etc.; and the higher order moment mainly describes the details of the image. The seven moment invariants are suitable for describing the overall shape of the objective function and are therefore widely used in edge extraction, image matching and target recognition ${ }^{[7]}$. At present, moments are widely used in many fields such as image matching, target recognition, shape feature analysis and so on.

The two-dimensional $(p+q)$ order of a digital image $f(x, y)$ is defined as:

$$
m_{p q}=\sum_{x} \sum_{y} x^{p} y^{q} f(x, y)
$$

Where, $p, q=0,1,2, \cdots$, the summation is performed on the value of all spatial coordinates across the image $x, y$.

The corresponding center distance is defined as:

$$
\mu_{p q}=\sum_{x} \sum_{y}(x-\bar{x})^{p}(y-\bar{y})^{q} f(x, y)
$$

Among them, $\bar{x}=\frac{m_{10}}{m_{00}}, \bar{y}=\frac{m_{01}}{m_{00}}$

Normalization $(p+q)$ The order center distance is defined as

$$
\eta_{p q}=\frac{\mu_{p q}}{\mu_{00}^{\gamma}}
$$

Among them, $\bar{x}=\frac{m_{10}}{m_{00}}, \bar{y}=\frac{m_{01}}{m_{00}}$

$$
\gamma=\frac{p+q}{2}+1 \text { Among them, } p+q=2,3, \cdots
$$

A set of seven 2D invariant moments that are not sensitive to translation, mirroring, and rotation can be derived from these formulas.

$$
\begin{gathered}
\phi_{1}=\eta_{20}+\eta_{02} \\
\phi_{2}=\left(\eta_{20}-\eta_{02}\right)^{2}+4 \eta_{11}^{2} \\
\phi_{3}=\left(\eta_{30}-3 \eta_{12}\right)^{2}+\left(3 \eta_{21}-\eta_{03}\right)^{2} \\
\phi_{4}=\left(\eta_{30}+\eta_{12}\right)^{2}+\left(\eta_{21}+\eta_{03}\right)^{2} \\
\phi_{5}=\left(\eta_{30}-3 \eta_{12}\right)\left(\eta_{30}+\eta_{12}\right)\left[\left(\eta_{30}+\eta_{12}\right)^{2}-3\left(\eta_{21}+\eta_{03}\right)^{2}\right]+\left(3 \eta_{21}-\eta_{03}\right)\left(\eta_{21}+\eta_{03}\right) \\
{\left[3\left(\eta_{30}+\eta_{12}\right)^{2}-\left(\eta_{21}+\eta_{03}\right)^{2}\right]}
\end{gathered}
$$




$$
\begin{aligned}
\phi_{6}= & \left(\eta_{20}-\eta_{02}\right)\left[\left(\eta_{30}+\eta_{12}\right)^{2}-\left(\eta_{21}+\eta_{03}\right)^{2}\right]+4 \eta_{11}\left(\eta_{30}+\eta_{12}\right)\left(\eta_{21}+\eta_{03}\right) \\
\phi_{7}= & \left(3 \eta_{21}-\eta_{03}\right)\left(\eta_{30}+\eta_{12}\right)\left[\left(\eta_{30}+\eta_{12}\right)^{2}-3\left(\eta_{21}+\eta_{03}\right)^{2}\right]+\left(3 \eta_{12}-\eta_{30}\right)\left(\eta_{21}+\eta_{03}\right) \\
& {\left[3\left(\eta_{30}+\eta_{12}\right)^{2}-\left(\eta_{21}+\eta_{03}\right)^{2}\right] }
\end{aligned}
$$

\section{Hand image preprocessing}

\subsection{Image segmentation}

Before the feature is extracted, the collected hand image needs to be preprocessed to obtain the region of interest. Firstly, the threshold segmentation method is used to binarize and median the collected hand image to filter out the discrete noise points in the image. Because of the lack of positioning method, the position of the hand has a certain degree of freedom, and the segmentation error of the wrist part is large. Therefore, the shape feature of the palm and the finger is used as the classification feature. It is necessary to divide the wrist portion in the collected hand image ${ }^{[8]}$. Finally, the use of palm and finger images as a feature extraction object. Figure 1 shows the image before and after the division of the wrist.

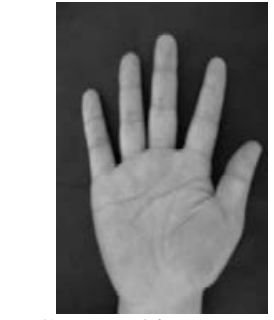

(a)Captured image

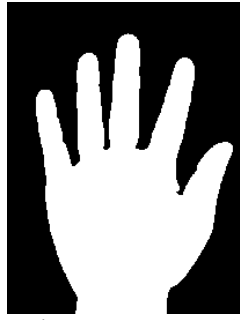

(b)Binary image

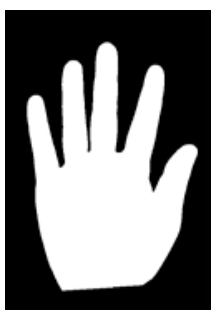

(c)after the division of the wrist image

Fig. 1 Original image and segmented hand image

\subsection{Normalized}

Since the Hu moment in the discrete case has rotation and translation invariance, but does not have scale invariance, it is necessary to normalize the image scale and translation in the pretreatment stage. The normalization step is:

1) Read the coordinates of the center of gravity of the sample image $(\bar{x}, \bar{y})$, The center of gravity of the image to be measured coordinates $\left(x_{0}, y_{0}\right)$, calculated

$$
\Delta x=\bar{x}-x_{0}, \Delta y=\bar{y}-y_{0}
$$

2) Translate the image to be measured:

$$
f(x, y)=f(x+\Delta x, y+\Delta y)
$$

3) Calculate the 0th moment of the sample image $m_{00}$ And the 0th moment of the image to be processed $\mathrm{m}_{00}^{\prime}$, According to scale $\alpha=\frac{\mathrm{m}_{00}}{\mathrm{~m}_{00}^{\prime}}$ Scale the image to be measured.

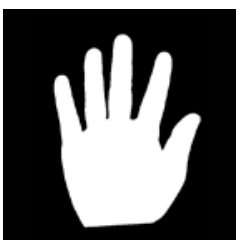

(a)

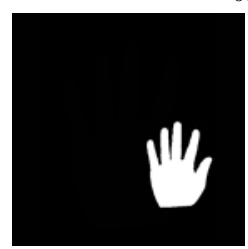

(b)

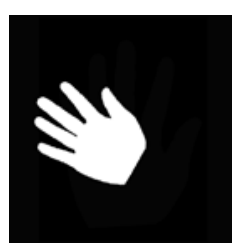

(c)

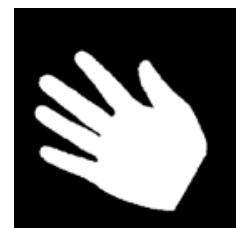

(d)

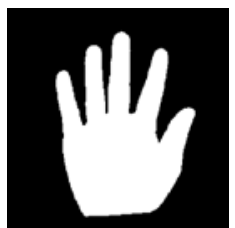

(e)

Fig.2 Normalized image 
The image before and after processing according to the above steps is shown in Fig. 2.

In Fig. 2, Fig. A is the image in the image library, b is the scaled-down image, $\mathrm{c}$ is the image after scaling, $d$ is the c normalized image, and e is the normalized image.

In order to compare the effect of normalization on the results, the HU moments of the five images in Fig. 2 are calculated respectively. The results are shown in Fig. From the results, it can be concluded that the effect of image scaling on feature extraction is obvious, and the characteristic curve extracted after normalization coincides with the original image. And the normalization step is essential for subsequent feature extraction and recognition.

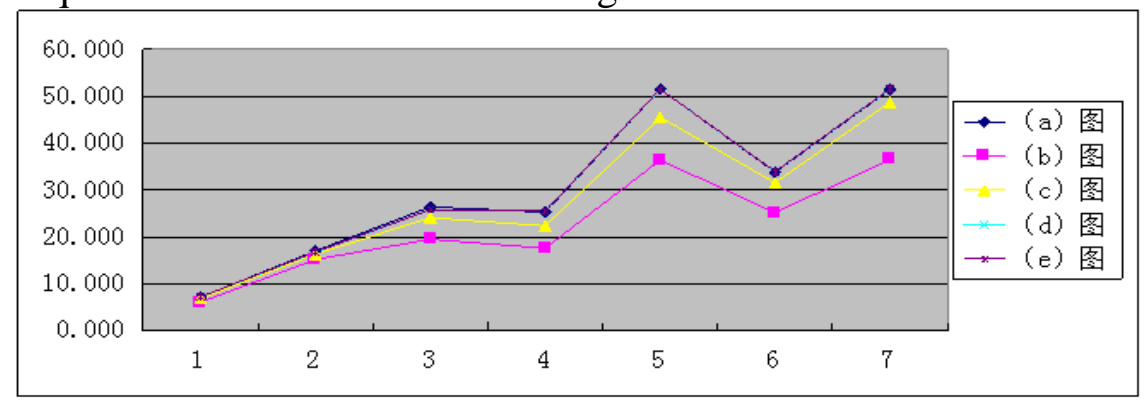

Fig.3 Eigenvalues of original image and normalized images

\section{Hand image moment feature extraction}

The traditional method based on the hand feature vector uses the geometry of the hand as the matching feature. The algorithm is simple to match the speed, but it is necessary to set the fixed bolt on the collection station to limit the placement of the hand, and the user needs to cooperate The The use of the moment feature representation image is an algorithm based on statistical analysis, which describes the overall shape feature of the image. In reference 9, it is proposed to apply the zernike moments based on orthogonal polynomials to hand recognition, which can construct any high order moments Variables, have good performance in terms of noise sensitivity and information redundancy, but there is no doubt that higher-order moments of computations increase computational complexity and are not applicable in applications where identification time is required ${ }^{[9]}$. The method proposed in this paper only need to fix the camera position after the collection of hand shape. Because the position of the hand has a large degree of freedom (small angle rotation or translation), so the extracted image features must be on the rotation or translation with invariance, HU moment to meet this requirement, the following we collected the same person Hand shape, and the different angles of rotation, translation and scaling of different scales, and calculate the HU moment results. Figure 4 shows the result of the rotation and translation of the same hand image. The first four images are the result of the original image translation, the second line of four images were respectively the original image by angle $15^{\circ}, 30^{\circ}, 45^{\circ}, 65^{\circ}$ rotation results, the third line of four images were different Scale scaling results after rotation.
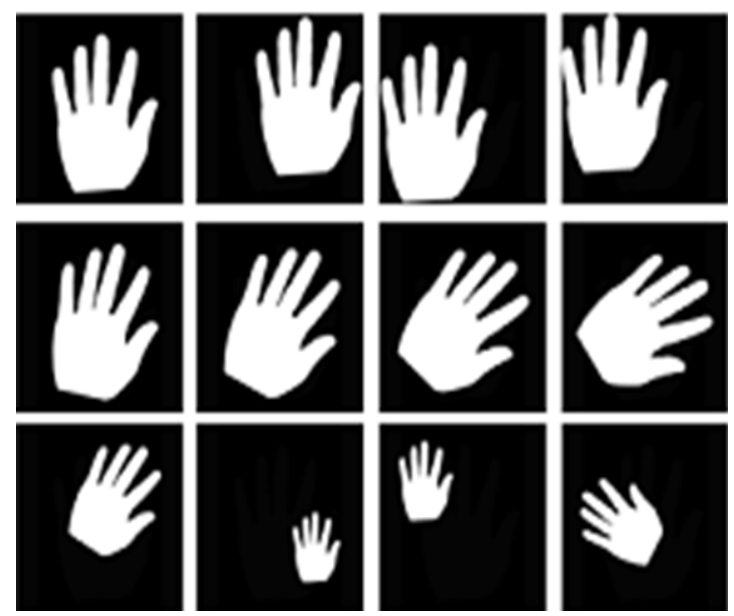

Fig.4 Original image and images after rotation, translation and scaling 
Table 1 lists the seven HU moments of the 12-image hand image in Figure 4 with a mean value of $\mu$ and a standard deviation of $\sigma, \sigma /(\mu \%)$ representing the percentage of each eigenvalue away from its mean, namely the discrete degree. The smaller the value of $\sigma /(\mu \%)$, the eigenvalue has a good rotation shift invariance ${ }^{[10]}$. From the data in the table, it can be concluded that the geometric invariant moments of the same hand shape have good invariance.

\section{Test results}

Identification of the problem to be resolved is that for a test to collect recognition hand shape, and hand box and enter the registration of the sample comparison, if the same subject, then the certification is successful, or need to re-capture or judged as not Belong to the same person. In this paper, the hand samples of 10 people in the laboratory were selected and collected 20 times in different time periods of the day. The position and angle of the hand were not restricted when collected. In this paper, the support vector machine typical two classification problem, that is, by collecting the hand shape and the image library user registration hand to compare, to determine whether the same person.

The traditional classification method only considers the fitting of the classifier to the training sample, aims at minimizing the classification error on the training set, and tries to improve the classifier in the untested test set by providing sufficient training samples for the training process The recognition rate. However, for a small set of training samples, it is not possible to ensure that a classifier that categorizes the training samples well categorizes the test samples ${ }^{[11]}$.

The support vector machine is based on the principle of minimizing the structural risk, that is, the minimization of training error and test error. It is widely used in pattern recognition (face recognition, character recognition, target automatic classification) and nonlinear system control. For the support vector machine classification, the first problem to be solved is the choice of kernel function. In this paper, we choose the radial basis function $(\mathrm{RBF})^{[12]}$ :

$$
K(x, y)=\exp \left(-\gamma\|x-y\|^{2}\right),
$$

Where the parameters $\gamma$ Size selection 0.5.

In order to evaluate the accuracy of the classification algorithm, we first selected 10100 sets of features from the same person in the 200 images collected, and then randomly selected 15 hand images as the test set in the image library to verify the classification of the algorithm. Sex. The classification results are shown in Table 1 . It can be seen that only the last one is randomly divided into the second category. Classification accuracy rate can reach 93.3\%, 15 images of the classification time of 0.87 seconds, classification accuracy and speed have reached a better effect.

\section{Conclusion}

In order to solve the inconvenient and unfriendly problem of the traditional hand recognition device, it is proposed to provide the user with the natural opening, the no locating bolt and the non-contact collecting hand shape image. The method of identity authentication. The geometric invariant moments of the normalized binary image are used as the feature matrix to solve the effect of the rotation of the hand during the acquisition and the scaling of the image due to the scale change to the recognition result.

In this paper, a hand-shaped library consisting of 200 sets of features is established, and seven geometric moment invariants are selected as eigenvalues. The support vector machine is used to train the sample images. Then, the training model is used to classify the randomly selected images. The accuracy rate can reach $93.3 \%$. The method proposed in this paper has high accuracy, low computational complexity, few features, easy transmission and storage, and can be applied to the development of embedded hand recognition device. 
Table 1 Invariant moment characteristics of hand shape images

\begin{tabular}{cccccccc}
\hline Image & \multicolumn{1}{c}{$\phi_{1}$} & $\phi_{2}$ & $\phi_{3}$ & $\phi_{4}$ & $\phi_{5}$ & $\phi_{6}$ & $\phi_{7}$ \\
& & & & & & & \\
\hline 1 & 7.1403 & 17.0060 & 26.3680 & 25.2830 & 51.5090 & 33.7960 & 51.4070 \\
2 & 7.1419 & 16.9930 & 26.4360 & 25.3580 & 51.5390 & 33.8600 & 51.6730 \\
3 & 7.1429 & 16.9590 & 26.2870 & 25.3860 & 51.4410 & 33.8690 & 51.7420 \\
4 & 7.1436 & 16.8910 & 26.0640 & 25.4240 & 51.3980 & 33.8740 & 51.6680 \\
5 & 7.1427 & 16.8180 & 25.9020 & 25.4130 & 51.2560 & 33.8230 & 51.6560 \\
6 & 7.1424 & 16.7430 & 25.8320 & 25.3810 & 51.2250 & 33.7550 & 51.4730 \\
7 & 7.1422 & 16.7860 & 26.0190 & 25.5350 & 51.6660 & 33.9460 & 51.6530 \\
8 & 7.1422 & 16.8600 & 25.9450 & 25.5890 & 52.4620 & 34.1570 & 51.4140 \\
9 & 7.1421 & 16.9170 & 26.0600 & 25.6010 & 51.8560 & 34.1000 & 51.7100 \\
10 & 7.1417 & 16.9860 & 25.8930 & 25.5110 & 54.7060 & 34.2290 & 51.2130 \\
11 & 7.1407 & 17.0070 & 26.0260 & 25.5130 & 51.9300 & 34.0640 & 51.4410 \\
12 & 7.1339 & 17.0890 & 26.1950 & 25.3970 & 53.0810 & 34.8090 & 51.2050 \\
$\mu$ & 7.1418 & 16.9299 & 26.0550 & 25.4349 & 52.1428 & 34.0065 & 51.4611 \\
$\sigma$ & 0.0028 & 0.1002 & 0.2085 & 0.1034 & 1.0398 & 0.2736 & 0.2714 \\
$\sigma /(\mu \%)$ & 0.0386 & 0.0059 & 0.0080 & 0.0041 & 0.0199 & 0.0080 & 0.0053 \\
\hline
\end{tabular}

Table 2 Classification result of the hand shape image

\begin{tabular}{crrrrrrrr}
\hline Image & $\phi_{1}$ & $\phi_{2}$ & $\phi_{3}$ & $\phi_{4}$ & $\phi_{5}$ & $\phi_{6}$ & $\phi_{7}$ & 类别 \\
\hline 1 & 7.1427 & 16.8180 & 25.9020 & 25.4130 & 51.2560 & 33.8230 & 51.6560 & 1 \\
2 & 6.8154 & 18.0790 & 23.7640 & 22.3540 & 45.4190 & 31.8310 & 47.5950 & 2 \\
3 & 7.1422 & 16.7860 & 26.0190 & 25.5350 & 51.6660 & 33.9460 & 51.6530 & 1 \\
4 & 7.1422 & 16.8600 & 25.9450 & 25.5890 & 52.4620 & 34.1570 & 51.4140 & 1 \\
5 & 7.0788 & 16.0992 & 25.2365 & 24.9677 & 51.8930 & 33.0208 & 50.0831 & 2 \\
6 & 6.2477 & 15.1710 & 20.2930 & 18.2430 & 37.6090 & 25.8880 & 38.3720 & 2 \\
7 & 7.1194 & 16.3246 & 24.0318 & 25.3875 & 51.4910 & 33.6278 & 50.1289 & 2 \\
8 & 7.1292 & 16.5126 & 24.3324 & 25.2624 & 50.5524 & 33.5781 & 50.2935 & 2 \\
9 & 7.1424 & 16.7430 & 25.8320 & 25.3810 & 51.2250 & 33.7550 & 51.4730 & 1 \\
10 & 6.0529 & 15.1800 & 19.4690 & 17.5630 & 36.3640 & 25.1650 & 36.4960 & 2 \\
11 & 7.1194 & 16.3246 & 24.0318 & 25.3875 & 51.4910 & 33.6278 & 50.1289 & 2 \\
12 & 7.1292 & 16.5126 & 24.3324 & 25.2624 & 50.5524 & 33.5781 & 50.2935 & 2 \\
13 & 7.0907 & 15.8063 & 26.5279 & 25.3051 & 53.8028 & 33.2082 & 51.2244 & 2 \\
14 & 7.0867 & 15.9037 & 26.4047 & 25.0624 & 52.4844 & 33.0178 & 50.8133 & 2 \\
15 & 7.1339 & 17.0890 & 26.1950 & 25.3970 & 53.0810 & 34.8090 & 51.2050 & $2 *$ \\
\hline
\end{tabular}

\section{References}

[1] Nicolae Dta. A survey of biometric technology based on hand shape[J], Pattern Recognition, 2009, 42: 2797-2806.

[2] Sang Hai-feng, Zhao Yun, Yuan Wei-qi, ChenJing. Multi-biological features recognition based on natural open of hand[J]. Chinese Journal of Scientific Instrument, 2011, 32(11): 2549-2556. (in Chinese)

[3] Yuan Wei-qi, Dong Qian. Hand Shape Verification Method Based on Hand Geometry Feature[J]. ACTA OPTICA SINICA, 2010, 30(10): 2994-2999. (in Chinese) 
[4] Yuan Wei-qi, Zhu Chun-yan, Ke Li. Hand shape identification method based on finger relative length[J]. Journal of Optoelectronics•Laser. 2009, 20(5): 685-689.

[5] Yuan Wei-qi, Dong Qian, Sang Hai-feng. Hand shape contour tracking method based on directional gradient extremum[J]. Optics and Precision Engineering, 2010, 18(7): 1675-1683.

[6] Gu Li, Zhuang Zhen-quan, Wan Shu-chao, Cai Wei. A Method of Hand Shape Verification Based on Template Matching Rules[J]. Computer Engineering and Applications, 2005, 6: 85-88.

[7] Gholamreza Amayeh, George Bebis, Ali Erol, Mircea Nicolescu. Peg-Free Hand Shape Verification Using High Order Zernike Moments[J]. IEEE CVPRW, 2006.

[8] Jiang Lu, Zhang Pin-zheng, Shu Hua-zhong. Moment application to human facial expression recognition[J]. JOURNAL OF SOUTHEAST UNIVERSITY (Nature Science Edition), 2004, 34(4): 557-560.

[9] Zhu Xu-feng, Ma Cai-wen, Liu Bo. Aircraft recognition scheme based on feature fusion and support vector machine[J]. Journal of Optoelectronics•Laser, 2011, 22(11): 1710-1713.

[10] S. Bourennane, C. Fossati. Comparison of shape descriptors for hand posture recognition in video[J]. SIViP, 2012(6):147-157.

[11] Ryszard S. Choras. Hand Shape and Hand Gesture Recognition[J]. IEEE ISIEA, 2009: 145-149.

[12] Li Ming-li, Hou Yu-qing, Cheng Tao, Chang Zhe, Liu Li-ning. One image shape retrieval method based on generalized moment invariants of the brink[J], Journal of Northwest University(Nature Science Edition), 2009, 39(4):545-548. 